EPJ Web of Conferences 16, 07001 (2011)

DOI: $10.1051 /$ epjconf/20111607001

(C) Owned by the authors, published by EDP Sciences, 2011

\title{
New technologies for exoplanet detection with mid-IR interferometers
}

\author{
P.R. Lawson ${ }^{1}$, a , O.P. Lay ${ }^{1}$, S.R. Martin ${ }^{1}$, R.D. Peters ${ }^{1}$, A.J. Booth ${ }^{1,2}$, \\ R.O. Gappinger ${ }^{1}$, A. Ksendzov ${ }^{1}$ and D.P. Scharf ${ }^{1}$ \\ ${ }^{1}$ Jet Propulsion Laboratory, California Institute of Technology, 4800 Oak Grove Drive, \\ Pasadena, CA 91109, USA \\ ${ }^{2}$ Sigma Space Corp., 4801 Forbes BIvd., Lanham, MD 20706, USA
}

\begin{abstract}
This paper provides an overview of technology development for the Terrestrial Planet Finder Interferometer (TPF-I). TPF-I is a mid-infrared space interferometer being designed with the capability of detecting Earth-like planets in the habitable zones around nearby stars.
\end{abstract}

\section{INTRODUCTION AND OVERVIEW}

The Terrestrial Planet Finder Interferometer (TPF-I) is a concept for a NASA mission to detect and characterize the mid-infrared spectra of Earth-like exoplanets [1,2]. The mission has been developed in close collaboration with the European Space Agency over the past decade as well as with members of the Darwin proposal team [3]. The primary objective is to find evidence of biological activity on planets around nearby stars.

The mid-infrared waveband of 6-20 $\mu \mathrm{m}$ provides a favorable planet/star contrast and key biosignatures, including those of water vapor, methane, oxygen, and carbon dioxide.

The designs for TPF-I and Darwin have been based on the use of formation-flying arrays of telescopes, with interferometer baselines of $40 \mathrm{~m}$ or more providing the angular resolution necessary to search for Earth-like planets around $\sim 150$ stars.

The relative positions of the telescopes need only be controlled to tens of centimeters, because delay lines are used within the beam combiner to provide nanometer-level relative phasing of the incoming starlight. This design is a compelling choice for a mid-infrared observatory; the formation flying baselines provide unrivaled angular resolution. This is vital for unambiguous orbit determination, the ability to distinguish multiple planets, and provides discrimination against structure in an exozodiacal disk. The angular resolution also provides a small inner working angle, giving access to a very broad range of target stars. In this regard, TPF-I and Darwin far exceed the predicted capability of other proposed planet-finding missions [4].

The major technology challenges for the mission include (1) the design of guidance, navigation and control for a formation flying array; (2) cryogenic designs for passive cooling of the optics and active cooling of detectors, and (3) the implementation of interferometer systems for starlight suppression and planet detection. The overall mission risk is dominated by the reliance on a coordinated use of multiple spacecraft. Aspects of cryogenic design and cooling are being addressed by missions such

ae-mail: Peter.R.Lawson@jpl.nasa.gov

This is an Open Access article distributed under the terms of the Creative Commons Attribution-Noncommercial License 3.0, which permits unrestricted use, distribution, and reproduction in any noncommercial medium, provided the original work is properly cited. 


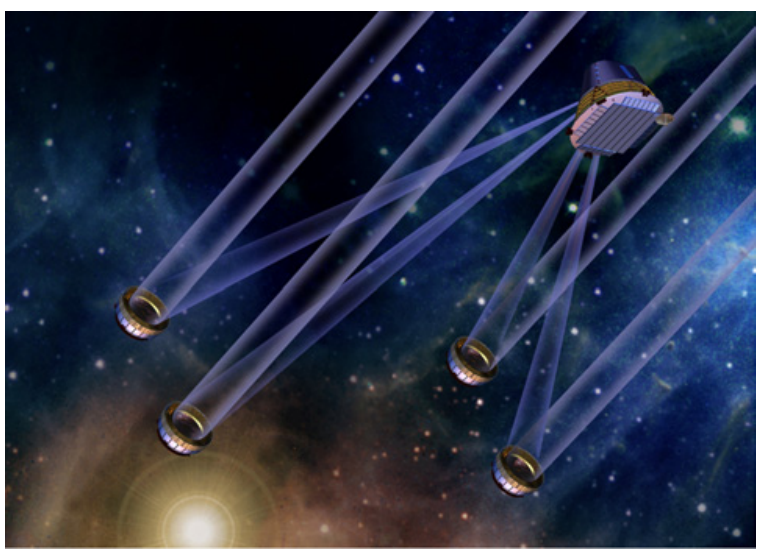

Figure 1. Artist's concept of the TPF/Darwin mid-infrared formation flying array. (Spacecraft image courtesy of T. Herbst, MPIA).

as Herschel and the James Webb Space Telescope; these topics are not discussed here. This paper is primarily devoted to a discussion of technology progress in the areas of starlight suppression and formation flying. Technology highlights of the past few years include the following:

1. The adoption of the Emma X-Array by both the TPF-I Project and the Darwin proposal team as the baseline mission design for TPF-I and Darwin [3].

2. Demonstration of agreement between independent performance models of the Emma X-Array [5], and the completion of a comprehensive target star catalog [6].

3. Milestone \#1: the compensation of intensity and phase demonstrated by the Adaptive Nuller testbed. Intensity was compensated to within $0.2 \%$ and phase to within $5 \mathrm{~nm}$ rms across a $3-\mu \mathrm{m}$ band centered at $10-\mu \mathrm{m}$ [7]. This is consistent with the flight requirement of the mission.

4. Milestone \#2: a demonstration of precision formation maneuvers in a ground-based robotic testbed, with performance traceability to flight [8].

5. Milestone \#3: a demonstration of broadband nulling at the flight requirements of $1.0 \times 10^{-5}$ [9]. A $34 \%$ bandwidth was used centered at a wavelength of $10 \mu \mathrm{m}$. Monochromatic nulls of $5.0 \times 10^{-7}$ have also been demonstrated [10].

6. Milestone \#4: Laboratory demonstration of the detection of a planet signal 1,000,000 times fainter than a star while using array rotation, chopping, and averaging [11].

7. Demonstrations of rendezvous and docking maneuvers in space by DARPA's Orbital Express and ESA's Autonomous Transfer Vehicle, Jules Verne.

The results of most testbed work for TPF-I accomplished to date have now been submitted for publication in refereed journals. In 2009-2010 the focus of continuing work will be on the broadband demonstration of instability noise suppression using the Planet Detection Testbed. This will be Milestone \#5. Continued progress in formation flying is anticipated through in-space testing over the next five years with missions sponsored by ESA and its European partners.

\section{TECHNOLOGY FOR STARLIGHT SUPPRESSION}

A mid-infrared interferometer must suppress starlight by a factor of approximately 1 part in 100,000 and integrate to extract planet spectra despite a background (due to local and exozodiacal dust) that may still be 100 times brighter than the planet light. 


\subsection{Mid-IR spatial filter development}

Spatial filters are an essential technology for nulling interferometry. They significantly reduce the optical aberrations in wavefronts, making extremely deep nulls possible. The most basic form of a spatial filter is a simple pinhole, and pinholes have indeed been used to achieve the deepest laser nulls so far at mid-infrared wavelengths. However, pinholes only operate well over a narrow bandwidth and so are ill-suited for broadband spatial filtering for science instruments. In addition, they do not reject very low spatial-frequency wavefront aberrations. The development of improved broadband techniques for spatial filtering at mid-infrared wavelengths may be crucial to the success of TPF-I, and has been a focus of research at JPL. Although fiber optics at near-infrared wavelengths are extensively used by the telecommunications industry, low-loss, mid-IR, single- mode spatial filters are not yet commercially available. The goal of this work has been to develop single-mode spatial filters with a throughput of $50 \%$ or better with a modal suppression of $25 \mathrm{~dB}$ for non-fundamental modes.

Spatial filters may be implemented in a variety of ways, including single-mode fiber-optics made from chalcogenide or silver halide glasses, metalized waveguide structures micro-machined in silicon, or through the use of photonic crystal fibers. By promoting the parallel development of various spatial filter technologies, it was hoped that we would demonstrate the necessary spatial filter performance in the 6-20 $\mu \mathrm{m}$ spectrum using no more than two technology types. The development of mid-infrared spatial filters was funded by TPF-I from 2003 through 2008. The performance of the single-mode filters that were developed under contract with TPF-I were tested and characterized in-house at JPL. The scope of the work included prototypes of hollow waveguide filters designed by Christopher Walker at the University of Arizona; completed chalcogenide fibers designed by Dr. Jas Sanghera at the Naval Research Laboratory (NRL); and completed silver halide fibers designed by Prof. Abraham Katzir at Tel Aviv University (TAU) in Israel.

The results of this research have been published by Ksendzov et al. [12, 13]. The fibers that have been developed for TPF-I represent the state of the art. About 16 mid-infrared single-mode fibers were delivered and tested at JPL, showing excellent single-mode behavior at $10 \mu \mathrm{m}$.

The 20-cm-long chalcogenide fibers developed by the Naval Research Laboratory were shown to demonstrate $30 \mathrm{~dB}$ rejection (a factor of 1000) of higher order modes and have an efficiency of $40 \%$, accounting for both throughput and Fresnel losses. The transmission losses were measured at $8 \mathrm{~dB} / \mathrm{m}$, and the fibers are usable up to a wavelength of about $11 \mu \mathrm{m}$. The chalcogenide fibers developed at the Naval Research Laboratory were used in the Achromatic Nulling Testbed and continue to be used in the Adaptive Nuller testbed, shown in Figure 2.

The $10-20 \mathrm{~cm}$ long silver halide fibers that were developed by Tel Aviv University were shown to demonstrate $42 \mathrm{~dB}$ rejection (a factor of 16,000) of higher order modes with transmission losses of $12 \mathrm{~dB} / \mathrm{m}$. This high rejection of higher-order modes was accomplished with the addition of aperturing of the output of the fibers, made possible by the physically large diameter of the fiber cladding. Silver halide fibers should in principle be usable up to a wavelength of about $18 \mu \mathrm{m}$, although the laboratory tests at JPL were conducted only at $10 \mu \mathrm{m}$. This is the first time silver halide fibers were shown to have single-mode behavior.

An important step in future work will be to demonstrate single-mode performance over the entire wavelength band that TPF-I will use $(6-20 \mu \mathrm{m})$. To date, experiments that test single-mode behavior have been limited to a narrow wavelength range near $10 \mu \mathrm{m}$. The spatial filtering capabilities of photonic crystal fibers should be investigated for use at mid-infrared wavelengths, because of the improved throughput that they may provide. Moreover, the suitability of these fibers for use in a cryogenic environment should be tested.

\subsection{Achromatic phase shifter studies}

The Achromatic Nulling Testbed (ANT) was developed to study achromatic phase shifting techniques to achieve broadband, dual-polarization, two-beam mid-infrared nulls. The two-beam nuller is the basic 


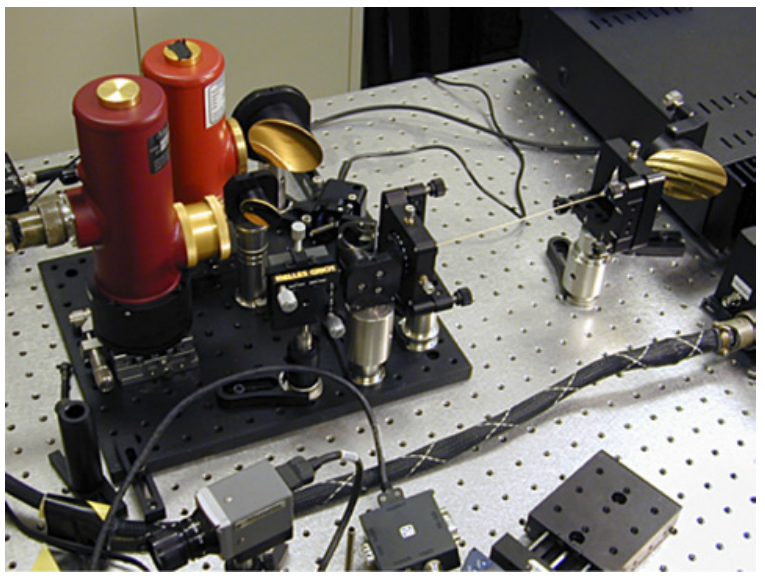

Figure 2. View of a chalcogenide glass fiber, in use within the Adaptive Nuller testbed. The fiber can be seen being fed by an off-axis parabola, to the right, prior to the spectrometer and single-pixel detector.

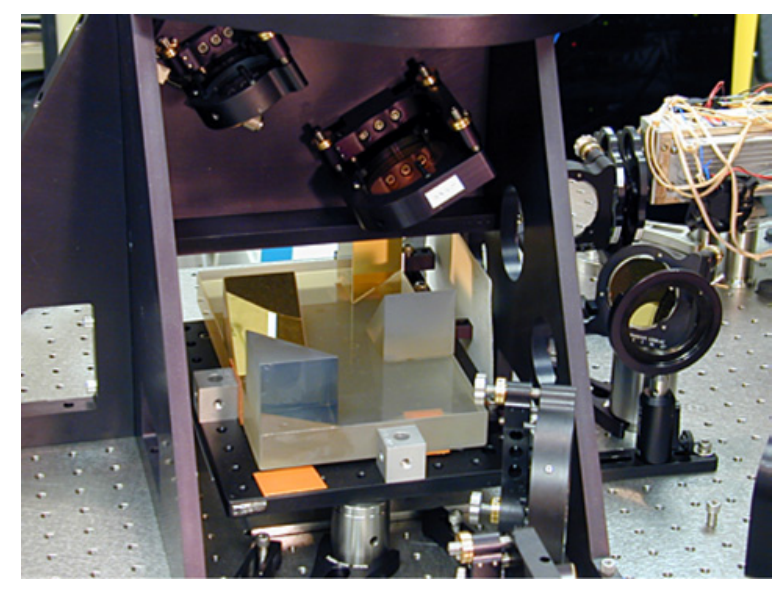

Figure 3. Side view of the periscope assembly of the Achromatic Nulling Testbed.

building block of all flight architectures that have been considered. Three approaches to achromatic phase shifting were investigated, with the aim of demonstrating, through one of the approaches, twobeam nulling to a level of 1 part in 100,000 with a $25 \%$ bandwidth in the $6-20 \mu \mathrm{m}$ range. A longerterm objective was the development of a cryogenic nulling interferometer that would meet the above requirements while operating at a temperature of $40 \mathrm{~K}$.

Three different methods of implementing achromatic phase shifts were investigated: (1) pairs of dispersive glass plates to introduce a wavelength-dependent phase delay; (2) A through-focus field-flip of the light in one arm of the interferometer; and (3) successive and opposing field-reversals on reflection off flat mirrors in a periscope arrangement. A close-up view of the periscope mirror is shown in Figure 3. These methods were tested in the same lab, on adjacent optical benches, using a common mid-infrared laser and white-light source.

The results of this work have been summarized by Gappinger et al. [14]. The most successful approach was the use of periscope mirrors, yielding an average rejection ratio of 51,000:1 at $20 \%$ bandwidth and 27,000:1 using a 25\% bandwidth. The through-focus approach yielded a rejection ratio 


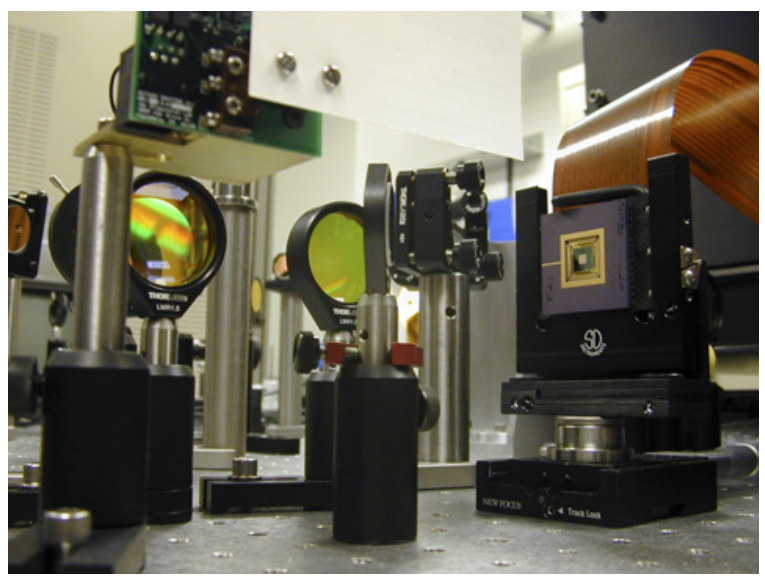

Figure 4. The Adaptive Nuller Testbed (AdN). The Adaptive Nuller demonstrated broadband nulling to the levels required for a flight mission. $1 \times 10^{-5}$ nulls were demonstrated using a bandwidth of $34 \%$ centered at a wavelength of $10 \mu \mathrm{m}$. This photo shows a closeup of the Adaptive Nuller's deformable mirror, which performs the phase and intensity compensation.

of $2000: 1$ with a $17 \%$ bandwidth. Pairs of glass plates provided a rejection ratio of $10,000: 1$ with a $25 \%$ bandwidth.

Of the methods of achromatic phase shifting that were tested by the ANT, the approach using the periscope mirrors produced the best results. Although these results fell short of the goal of 100, 000:1 at $25 \%$ bandwidth, this goal was nontheless attained by the Adaptive Nuller (next section). An adaptive nuller used in conjunction with a periscope phase shifter would also be a viable approach. The Achromatic Nulling Testbed was dismantled in January 2008.

\subsection{Adaptive nulling}

The Adaptive Nuller was designed to correct phase and intensity variations as a function of wavelength, in each of two linear polarizations. This should allow high performance nulling interferometry, while at the same time substantially relaxing the requirements on the interferometer's optical components.

The initial goal of the testbed was to demonstrate, in a $3-\mu \mathrm{m}$ band centered at a wavelength of $10 \mu \mathrm{m}$, the correction of the intensity difference to less than $0.2 \% \mathrm{rms}(1 \sigma)$ between the interferometer's arms, and at the same time correct the phase difference across the band to $\leq 5 \mathrm{~nm} \mathrm{rms}(1 \sigma)$. This overall correction is consistent with a null depth of $10^{-5}$ (1 part in 100,000) if all other sources of null degradation can be neglected.

The Adaptive Nuller uses a deformable mirror to adjust amplitude and phase independently in each of about 12 spectral channels. The incident beam is first split into its two linear polarization components, and is dispersed. The dispersed spectra are then imaged onto a line of pixels on a deformable mirror, so that the piston of each pixel independently adjusts the phase of each channel. Tilt in the orthogonal direction is independently adjusted, which shears the output pupil at that wavelength; this shear, in combination with an output stop, selectively reduces the intensity in that channel. The various component beams are recombined to yield an output beam that has been carefully tuned for intensity and phase in each polarization as a function of wavelength. A photograph of the deformable mirror used in testbed is shown in Figure 4.

The results of initial research on intensity and phase compensation were published by Peters et al. [7], based on results up until March/April 2007. This demonstrated phase compensation to better than $5 \mathrm{~nm}$ rms across the $8-12 \mu \mathrm{m}$ band and intensity compensation to better than $0.2 \%$. 

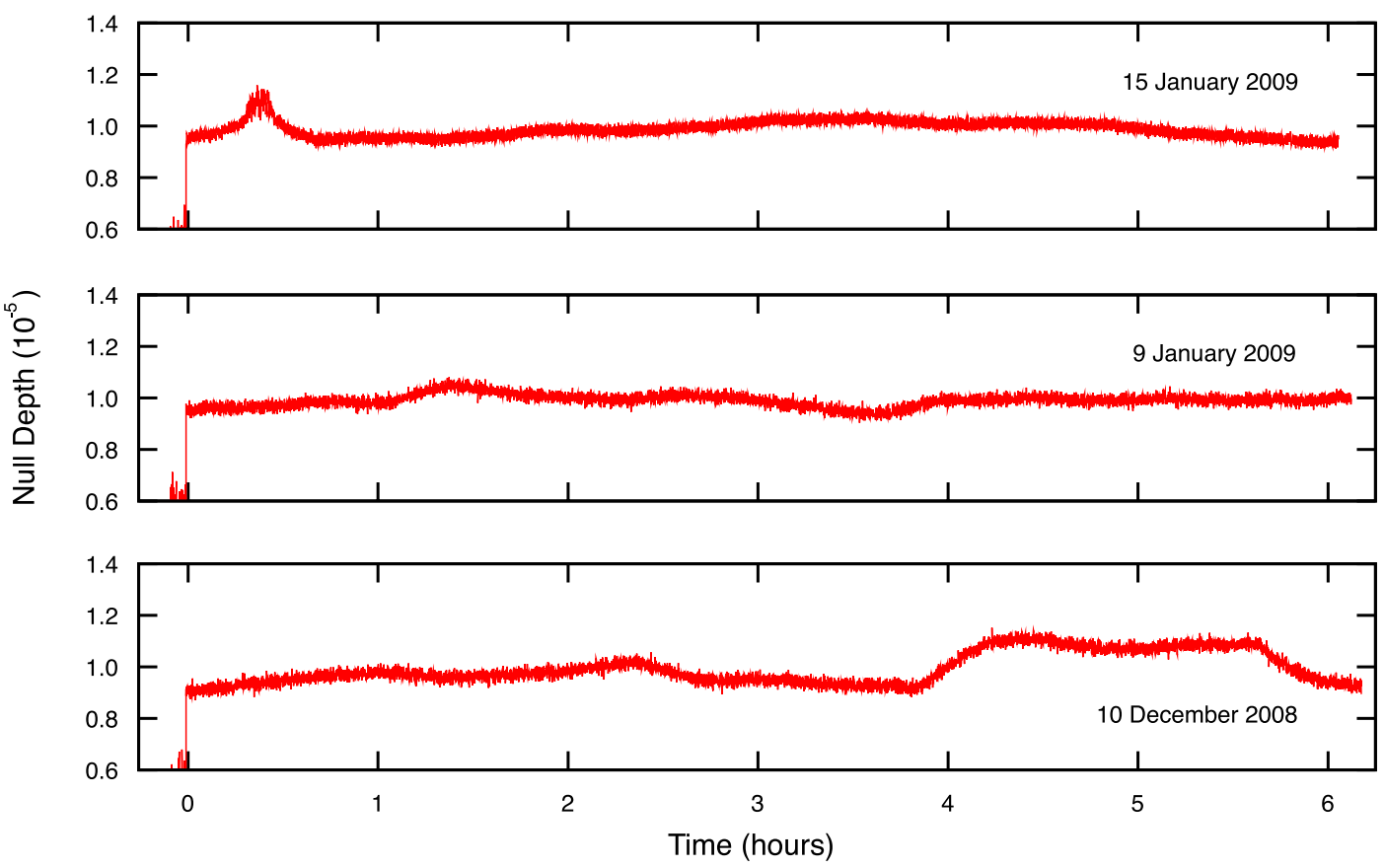

Figure 5. Broadband nulling data from the Adaptive Nuller. A total of 16 hours of data are shown from three separate 6-hour experiments. Each data set shows an average null depth better than $1 \times 10^{-5}$. This level of starlight suppression would dim the starlight so that it is fainter than the glow of a typical exozodiacal cloud that might surround a target system.

A subsequent goal was set to demonstrate broadband nulling at the levels required for a flight mission. The testbed was modified slightly to allow all the light across the band to be measured at a single pixel. Experiments were conducted to measure the null depth with time series spanning several hours.

In December 2008 and January 2009 Adaptive Nuller demonstrated a null depth better than $1.0 \times 10^{-5}(100,000: 1$ rejection ratio), over a bandwidth of $34 \%$, as shown in Figure 5 [9]. This is the deepest broadband null ever achieved by a mid-infrared nulling interferometer, and attains the TPF-I flight requirement. Long term plans include the possible design of a cryogenic version of this testbed.

\subsection{Planet Detection Testbed}

The Planet Detection Testbed, pictured in Figure 6, was developed to demonstrate the feasibility of four-beam nulling, the achievement of the required null stability, and the detection of faint planets using approaches similar to the ones contemplated for a flight-mission. The most promising architectures for a flight mission use synthesis imaging techniques with four-beam nulling interferometers and interferometric chopping to detect planets in the presence of a strong mid-infrared background. The flight mission would use a phase chopping technique to modulate a sensitivity/fringe pattern around the star. This modulation technique is in many ways similar to the use of a chopper wheel that allows the detection of infrared sources against a thermal background and/or drifting detector offsets. In this case the thermal background on the sky includes the local and exozodiacal light. The detected signal is the difference in the measured photon flux between the two chop states and this signal has both 


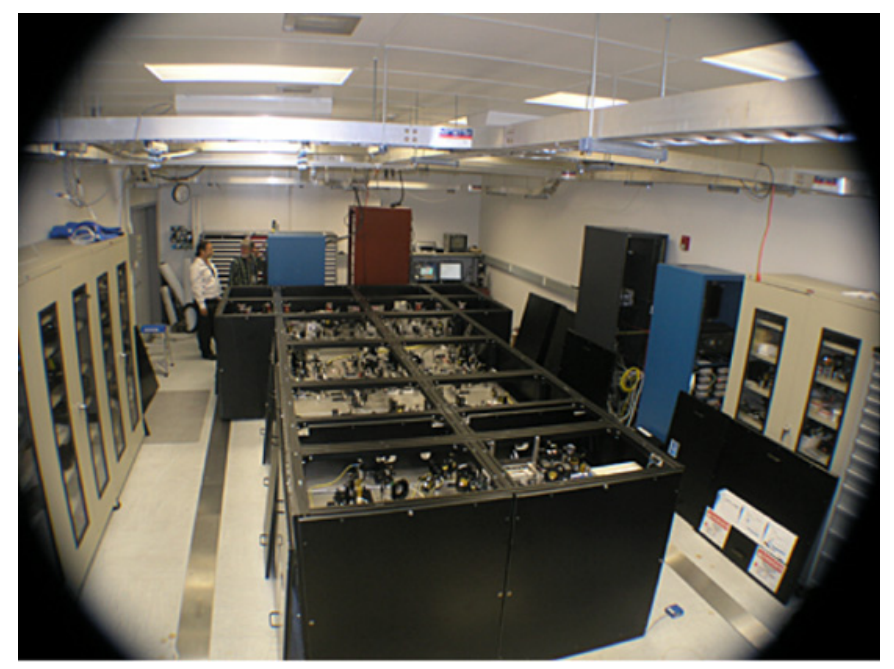

Figure 6. Overview of the Planet Detection Testbed (PDT). This testbed includes a 4-beam nulling beam combiner to emulate the system complexity of the Emma X-Array. In laboratory experiments with the PDT, planet signals a million times fainter than a star have been detected.

stochastic and systematic noise components. Integration over time reduces the stochastic components and good instrument stability is needed to minimize systematic components which may appear as low frequency fluctuations with timescales similar to a planet signal. Some of these systematic components can be removed by signal processing using expected correlations across the broadband light spectrum.

The PDT has the following main components: a star and planet source to generate a planet to be observed, a pair of nullers to null out the starlight, and a cross-combiner to allow modulation of the detected planet signal. To provide the necessary stability, the testbed has pointing and shear control systems, laser metrology systems and fringe trackers to maintain the phase on the star. The PDT combines simulated star and planet beams in pairs to produce four star/planet beams that enter the testbed as if detected through four separate telescopes. Delay lines are used for the planet light relative to the star-light to simulate the path delays that would be observed as the array rotates with respect to the star/planet system. Two beam pairs are nulled and then cross-combined. A $\pi$ phase shift is introduced into one of each beam pair by a combination of optical path differences in glass and air. The beam-pairs are chopped using a standard infrared approach and the difference in chop-states is recorded. The testbed is described in detail in the milestone report [11], and in a companion paper [15].

The PDT completed Milestone \#4 in October 2009. An example dataset is shown in Figure 7. The criteria for the milestone were as follows:

1. Detect a planet at a contrast of $10^{-6}$ relative to the star at a signal to noise ratio of $\geq 10$.

2. Show residual starlight suppression from phase chopping, averaging and rotation $\geq 100$.

3. The tests in (1) and (2) must each run for a total duration of 10,000 s and may include one or more planet rotations at timescales $\geq 2,000 \mathrm{~s}$.

4. The tests in (1) and (2) must be satisfied simultaneously on three separate occasions with at least 48 hours between each demonstration.

This constitutes a demonstration of three of the four main parts of the starlight suppression technique: deep interferometric nulling, phase chopping and formation rotation to modulate the planet. The fourth part is the extraction of a planet signal using a broadband spectral filtering technique [16], which will be the subject of Milestone \#5. The current plan is to attempt this additional milestone with the PDT in 2010-2011. 


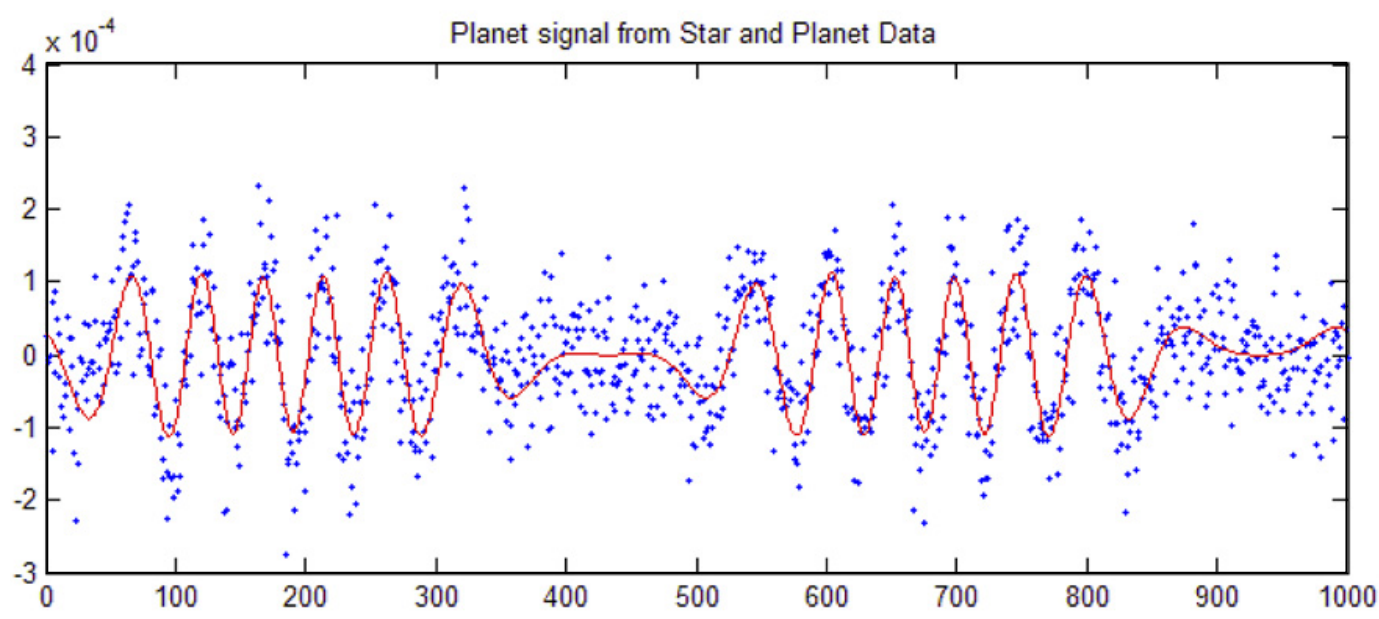

Figure 7. Planet signal detected with the Planet Detection Testbed, from experiments on 10 July 2009. The null depth was $7.4 \times 10^{-6}$, the planet/star contrast was $4.4 \times 10^{-7}$, and the planet is detected with a signal-to-noise of 16.4. The best-fit fringe pattern is shown for reference. Rotation, chopping, and averaging here provides an additional factor of 250 in noise suppression.

\section{TECHNOLOGY FOR FORMATION FLYING}

Technology for rendezvous and docking has been successfully tested in space by DARPA's Orbital Express mission in 2007, as well as ESA's Autonomous Transfer Vehicle, Jules Verne in 2008. Remarkable advances in technology have been demonstrated in recent years. Efforts in support of TPFI have been undertaken to demonstrate the guidance, navigation, and control necessary for precision formation maneuvers.

\subsection{Formation Control Testbed}

The Formation Control Testbed (FCT) was built to provide an end-to-end autonomous formation flying system in a ground-based laboratory. The FCT provides an environment for system-level demonstration and validation of formation control algorithms. The algorithms are validated using multiple floating test robots that emulate real spacecraft dynamics. The goal is to demonstrate algorithms for formation acquisition, maneuvering, fault-tolerant operations, as well as collision-avoidance maneuvering.

The FCT is comprised of two robots with flight-like hardware and dynamics, a precision flat floor for the robots to operate on, ceiling-mounted artificial stars for robot attitude sensing and navigation, and a "ground control" room for commanding the robots and receiving telemetry. The layout of the FCT emulates the environment of a formation of telescopes that is restricted to maneuvering in the same plane in space, normal to the direction of the target star. To be as flight-like as possible, each robot is equipped with a typical single-spacecraft attitude control suite of reaction wheels, gyros, and a star tracker. Thrusters are also available for attitude control. Each robot has a lower translational platform and an upper attitude platform. The attitude platform/spacecraft houses the avionics, actuators, sensors, inter-robot and "ground"-to-robot wireless communication antennae, and the spacecraft processors. The translational platform provides both translational and rotational degrees of freedom to the attitude platform via (i) linear air bearings that allow the entire robot to float freely on the flat floor, (ii) a vertical stage, and (iii) a spherical air bearing at the top of the vertical stage.

Each robot has six degrees-of-freedom: three in translation and three in rotation. The robots can translate wherever necessary on the flat floor; however, the pitch and roll axes of each robot's motion 
are limited to $\sim 30$ degrees (a physical limitation of the spherical air bearings). The vertical air bearings have a range of $\pm 25 \mathrm{~cm}$. The formation algorithms are designed for all six degrees-of-freedom.

In 2007 the Formation Control Testbed demonstrated its milestone for precision maneuvers using two robots, showing autonomous initialization, maneuvering, and operation in a collision free manner [8]. The key maneuver that was demonstrated was representative of TPF-I science observations. Repeated experiments with the robots demonstrated formation rotations through greater than 90 degrees of rotation at ten times the flight rotation rate while maintaining a relative position control to $5 \mathrm{~cm}$ rms. Although the achievable resolution in these experiments was limited by the noise environment of the laboratory, it was nonetheless demonstrated through modeling that in the relatively noise-free environment of space, the performance of these algorithms would exceed TPF-I flight requirements.

The next steps for technology maturation that could be done using the robots within the FCT include demonstrating new capabilities such as (1) reactive collision avoidance, (2) formation fault detection, and (3) autonomous reconfiguration and retargeting maneuvers. Using a real-time simulation environment we could also demonstrate the performance with full formation-flight complexity, that is with five interacting spacecraft showing synchronized rotations, autonomous reconfigurations, fault detection, and collision avoidance. Although NASA has no immediate plans to continue supporting formation flying research, national agencies in Europe are actively advancing the technology, with flight missions starting in 2010-2014.

In 2010 the Swedish Space Agency will launch the Prisma mission. This is primarily a rendezvous and docking mission, but will also test RF metrology that had been designed for Darwin. In 2012 ESA plans to launch Proba-3, which had been designed as a technology precursor to XEUS (now IXO); it will include optical metrology loops for sub-millimeter range control over a 30-m spacecraft separation.

The greatest advance in maturing technology for formation flying would certainly be to have a modest- scale technology mission devoted to verifying and validating guidance and control algorithms, and to further include the interferometric combination of starlight from separated platforms. A groundbased facility such as the FCT will continue to provide the means to test and improve real-time formation-flying algorithms as the technology matures, even while the technology is being proven in space.

\section{SUMMARY}

The technology program for the Terrestrial Planet Finder Interferometer is now close to achieving all of its milestones in starlight suppression. The basic component technology for starlight suppression at mid-infrared wavelengths is now at Technology Readiness Level (TRL) 4. TRL 5 requires testing in a relevant environment, which for TPF-I would be in vacuum at cryogenic temperatures near $40 \mathrm{~K}$. Most research so far has been undertaken in air at room temperature. However, sufficient progress has now been made that the greatest advance in this area would be to proceed to brass-board designs of already successful components and sub-systems.

The flight requirement for TPF/Darwin is to achieve a mean rejection ratio of 100,000:1 across the full science band of 6-20 $\mu \mathrm{m}$. Laboratory work with the Adaptive Nuller has demonstrated midinfrared rejection ratios at this level with bandwidths of $34 \%$ at a mean wavelength of 10 microns. These results show that the Adaptive Nuller is a viable solution to the design of achromatic phase shifters, and moreover that the chalcogenide single-mode fibers it uses are viable in the lower wavelength range of the science band $(6-12 \mu \mathrm{m})$. Adaptive nulling is straightforward to generalize over the full science band, and with continued support could be demonstrated within a cryogenic vacuum, bringing the technology to TRL 5.

The single-mode fibers used in the Adaptive Nuller are made of chalcogenide material and have been demonstrated to yield $25 \mathrm{~dB}$ or more rejection of higher-order spatial modes, thus meeting the flight requirements. Because this material becomes opaque at wavelengths above $12 \mu \mathrm{m}$, other materials must be used in the 12-20 $\mu \mathrm{m}$ wavelength band. Silver halide single-mode fibers have been demonstrated to 
meet flight requirements at $10 \mu \mathrm{m}$, and should also work well at longer wavelengths. Although this performance is sufficient for flight, it would be greatly advantageous to improve the throughput of these devices, to test them throughout the full wavelength range they are intended for, and to test them cryogenically. Spatial filter technology would then be at TRL 5. It would furthermore be advantageous to implement mid-infrared spatial filters and beam combiners using integrated optics, so as to reduce the risk associated with the complexity of the science instruments.

Demonstrations with the Planet Detection Testbed have shown that planet signals a million times fainter than a star can be recovered, and have also demonstrated the control algorithms for nulling while rotating, chopping, and averaging. Additional work is necessary to demonstrate the technique of spectral filtering to suppress instability noise.

The technology for broadband starlight suppression is now at a level mature enough to proceed with component brassboard designs, more ambitious system-level demonstrations, and extensive cryogenic testing.

In-space testing is necessary in the 2010-2020 decade to raise the TRL level of formation flying. This work should be done through an international collaboration and leverage both US and European expertise in separated-spacecraft rendezvous and docking.

This work was conducted at the Jet Propulsion Laboratory, California Institute of Technology, under contract with the National Aeronautics and Space Administration. Reference in this paper to any specific commercial product, process, or service by trade name, trademark, manufacturer, or otherwise, does not constitute or imply its endorsement by the United States Government or the Jet Propulsion Laboratory, California Institute of Technology.

Copyright 2009. All rights reserved.

\section{References}

[1] C.A. Beichman, N.J. Woolf, C.A. Lindensmith eds., The Terrestrial Planet Finder (TPF) (Jet Propulsion Laboratory Publications, Pasadena, California, JPL Pub. 99-3, May 1999)

[2] P.R. Lawson, O.P. Lay, S.R. Martin, R.D. Peters, R.O. Gappinger, A. Ksendzov, D.P. Scharf, A.J. Booth, C.A. Beichman, E. Serabyn et al., Terrestrial Planet Finder Interferometer: 2007-2008 progress and plans, in Optical and Infrared Interferometry, edited by M. Schöller, W.C. Danchi, F. Delplancke (2008), Vol. 7013 of Proc. SPIE, p. 7013N

[3] C.S. Cockell, A. Léger, M. Fridlund, T. Herbst, L. Kaltenegger, O. Absil, C. Beichman, W. Benz, M. Blanc, A. Brack et al., Astrobiology 9, 1 (2009)

[4] O.P. Lay, S.R. Martin, S.L. Hunyadi, Planet-finding performance of the TPF-I Emma architecture, in Techniques and Instrumentation for Detection of Exoplanets III, edited by D.R. Coulter (2007), Vol. 6693 of Proc. SPIE, p. 66930A

[5] D. Defrère, O. Lay, R. den Hartog, O. Absil, Earth-like planets: science performance predictions for future nulling interferometry missions, in Optical and Infrared Interferometry, edited by M. Schöller, W.C. Danchi, F. Delplancke (2008), Vol. 7013 of Proc. SPIE, p. 701321

[6] P.R. Lawson, O.P. Lay, K.J. Johnston, C.A. Beichman eds., The Terrestrial Planet Finder Science Working Group Report (Jet Propulsion Laboratory, Pasadena, California, JPL Pub. 07-1, March 2007)

[7] R.D. Peters, O.P. Lay, M. Jeganathan, Appl. Opt. 47, 3920 (2008)

[8] D.P. Scharf, J.A. Keim, F.Y. Hadaegh, IEEE Systems J. 4, in press (2010)

[9] R.D. Peters, O.P. Lay, P.R. Lawson, Pub. Astron. Soc. Pac. 122, in press (2010)

[10] A.J. Booth, S.R. Martin, F.M. Loya, Pub. Astron. Soc. Pac. 122, submitted (2010)

[11] S.R. Martin, A.J. Booth, O.P. Lay, P.R. Lawson, Exoplanet Interferometry Technology Milestone \#4 Report: Planet Detection Demonstration (Jet Propulsion Laboratory Publications, Pasadena, California, JPL Document 63261, 19 October 2009) 
Research, Science and Technology of Brown Dwarfs and Exoplanets

[12] A. Ksendzov, O. Lay, S. Martin, J.S. Sanghera, L.E. Busse, W.H. Kim, P.C. Pureza, V.Q. Nguyen, Appl. Opt. 46, 7957 (2007)

[13] A. Ksendzov, T. Lewi, O.P. Lay, S.R. Martin, R.O. Gappinger, P.R. Lawson, R.D. Peters, S. Shalem, A. Tsun, A. Katzir, Appl. Opt. 47, 5728 (2008)

[14] R.O. Gappinger, R.T. Diaz, A. Ksendzov, P.R. Lawson, O.P. Lay, K.M. Liewer, F.M. Loya, S.R. Martin, E. Serabyn, J.K. Wallace, Appl. Opt. 48, 868 (2009)

[15] A.J. Booth, S.R. Martin, F.M. Loya, Exoplanet Exploration Program, Planet Detection Testbed: Latest results of planet light detection in the presence of starlight, in Optical and Infrared Interferometry, edited by M. Schöller, W.C. Danchi, F. Delplancke (2008), Vol. 7013 of Proc. SPIE, p. 701320

[16] O.P. Lay, Removing instability noise in nulling interferometers, in Advances in Stellar Interferometry, edited by J.D. Monnier, M. Schöller, W.C. Danchi (2006), Vol. 6268 of Proc. SPIE, p. 62681A 